\title{
Using GIS for Modeling a Spatial DSS for Industrial Pollution in Egypt
}

\author{
Christina Albert Rayed
}

Computer and Information System dept. Sadat Academy for Management Science Cairo, Egypt

\begin{abstract}
Geographic Information Systems (GIS) have become an effective tool for decision support. Spatial Decision Support System (SDSS) is a relatively new field developed based on Geographic In formation System (GIS) and Decision Support System(DSS). SDSS will be an important component of DSS applications in future. This trend will be driven by the relevance of spatial information as a component of the information needed for a wide range of decisions. This class of DSS will make an important contribution, not because of its use of the latest technology, but because it will allow decision makers incorporate a spatial dimension in their decision making. So Spatial Decision Support Systems (SDSS) are decision support systems where spatial properties of the data to be analyzed play a major role in decision making special in many sectors. Maps and geographic features can be used to show decision related information and relationship between objects to solve important problems like in spreading diseases and industrial pollution.
\end{abstract}

Keywords Geographical Information Systems (GIS), Decision Support System(DSS), Spatial Decision Support Systems (SDSS), Industrial Pollution

\section{Introduction}

The continuing development of DSS applications requires that new technologies be exploited to allow new classes of decision be supported. This paper discusses the use of a Geographic Information System(GIS) as a Decision Support System (DSS) generator to create Spatial Decision Support Systems (SDSS). A geographic information system is a system designed to capture, store, manipulate, analyze, manage, and present all types of geographical data. The_GIS is sometimes used for geographical information science or geospatial information studies to refer to the academic discipline or career of working with geographic information systems[1]. Geographic information science is the science underlying geographic concepts, applications, and systems[2]. The GIS implementation stage consisted of the creation of a relational database, including a complete set of georeferenced data, and the elaboration of user interface for spatial analysis using GIS facilities in order to establish a Decision Support System (DSS). The current development of how to use models is shifting from individual modeling techniques toward finding the synergies between modeling techniques and combining them, to solve real world problems [3]. Integrated computer mode ling enables far more variables to be taken into account than is possible otherwise.

* Corresponding author:

christinalbert2004@yahoo.com (Christina Albert Rayed)

Published online at http://journal.sapub.org/ajgis

Copyright (C) 2012 Scientific \& Academic Publishing. All Rights Reserved
The choice for dynamic modeling has been made because important driving forces and processes change over time, and actions and developments that have taken place are very often not reversible; indicating a path-dependency of developments [4]. GIS allows the integration of geographical referenced data, together with local knowledge in relational databases to accurately display complex interactions in simple formats[5]. By combining geographic data from a wide range of sources, managers can quickly assemble custom maps to expedite a range of management activities. This process of creating new GIS outputs from existing data is referred to as modeling a spatial decision support system. Such modeling converts existing datasets into new datasets by applying Decision Support System(DSS) Models, Expert System (ES) and Artificial Intelligence (AI). This combination of data can create a model output that helps answer questions posed with spatial relevance. Typically, this is not modeling in terms of integrated model. GIS data presentation is of considerable value when spatial and visual representation is important.

The evolution of the DSS may be divided into four generations: the first DSS generation focused on data; the second DSS generation focused on improving the user interface; the third DSS generation focused on models and the fourth, the present-day generation, was obtained by introducing new analytical web-based applications [6]. As a short conclusion, the Decision Support Systems belong to a multid isciplinary environment, including database research, artificial intelligence, human-co mputer interaction, simulati on methods, software engineering and telecommunications. 
Thus, the concept of Decision Support Systems is an almost established concept, but which is still growing due to the integration (incorporation) of several individual and relatively newer technologies (object orientation, expert systems, advanced communications), from which it extracts new valences and strengths. Concurrently, the vitality of the concept is stimulated by the growing tendency of integrating processes and functions with all industrial systems, environment management systems, etc. The systems that used to provide support in the decision process have been named by specialists Decision Support Systems or Decision Management Systems. Recently, terms such as artificial intelligence, data mining, on-line analytical processing, and knowledge management have been used for systems whose objective was to inform and assist managers in the decision process.

Spatial Decision Support Systems (SDSS) are decision support systems where spatial properties of the data to be analyzed play a major role in decision making special in health sector. Maps and geographic features can be used to show decision related information and relationship between objects to solve important problems like in the relation between some industrial pollution and some diseases. Based on the understanding that SDSS is a kind of information system capable of providing spatial decision making schemes to decision-makers, we present our paper to helping decision makers in Egypt. So SDSS is an interactive, computer-based system designed to support a user or group of users in achieving a higher effectiveness of decision making while solving a semi-structured spatial problem[7]. It is designed to assist the spatial planner with guidance in making land use decisions. SDSS typically uses a variety of spatial and nonspatial information, like data on land use, transportation, water management, demographics, agriculture, climate or emp loy ment.

\section{Spatial Decision Support System}

SDSS typically uses a variety of spatial and nonspatial information, like data on land use, transportation, water management, demographics, agriculture, climate or emp loyment. By using two or more known points in history the models can be calibrated and then projections into the future can be made to analyze different spatial policy options. Using these techniques spatial planners can investigate the effects of different scenarios, and provide information to make informed decisions. To allow the user to easily adapt the system to deal with possible intervention possibilities an interface allows for simple modification to be made. Managers use computerized decision support systems for accessing important information, the principles of decision making, modeling and how business intelligence tools are used to support decision making[8].

GIS database management systems (DBMSs) are designed to support cartographic display and spatial query. Database of an SDSS must support cartographic display, spatial query and analytical modeling by integrating three types of data: 1. locational (spatial primitives such as coordinates and chains) 2. Topological (attribute-bearing objects, e.g. points, nodes and lines, and relationships between them) 3. Thematic (attributes of the topological objects, including population, elevation, and vegetation).

Database must permit the user to construct and exploit complex spatial relations between all three types of data at a variety of scales, degrees of resolution and levels of aggregation. Database management systems found in many GIS use the relational data model. However, alternative data models have proved effective in applications of DSS. The extended network model is an enhanced form of the network model and is effective for representing the links and nodes of transportation networks. Transportation networks are a popular base for developing SDSS because of the importance of applications for site selection and the abundance of methods of analysis.

Multi-Criteria Decision Analysis methods support decision-makers in analyzing a set of alternative spatial solutions, such as the most likely ecological habitat for restoration, against multiple criteria, such as vegetation cover or roads. MCDA uses decision rules to aggregate the criteria, which allows the alternative solutions to be ranked or prioritized[9].

\section{Industrial Pollution in Egypt}

\section{A. Water pollution}

The protection of water from pollution represents another important priority. The EEAA in this respect, Law 4/1994 for the Environment places an emphasis on the protection of the coastal wasters and the marine environment, complementing Law 48/1982 for the protection of the River Nile[10]. The lines of action in this regard encompass water quality monitoring activities and initiatives, as well as pollution abatement and mitigation efforts[11].

Certain types of significantly polluting industries have a specific geographical distribution, such as the cement, the iron and steel, and the coke and chemical industries in Cairo; textile, food, oil and soap industries in Alexandria and the Delta region; and sugar in Upper Egypt. During the last few years, significant attention has been given to the protection of the Nile from pollution. The focus is on industrial establishments, since industrial wastewater is the major contributor to Nile pollution. Underway is the finalization of the 2001 study, which covers the whole stretch of the river in Egypt, from Aswan to the Mediterranean[12].

The negative pressure generated increases the likelihood of infiltration of ground water and sewage into the pipes. Moreover, the tanks are open for contamination by atmospheric depositions, birds and animals[13].

In addition, chemical contamination of the drinking water especially in Cairo and other large Egyptian cities are not to be neglected. Chlorine, carcinogenic pesticides, heavy metals especially lead all been detected in varying levels in 
our drinking water. In Cairo, however, the main health hazard from drinking water is lead contamination. Microbiological contamination of drinking water in some suburban areas and rural communities in Egypt is not to be neglected. Potent water supplies are still not available in a major sector of non-urban areas of Egypt including a large section of Egyptian population[14].

\section{B. Pesticides Exposure and Reproductive Health}

Organochlorines including endrin, dieldrin, lindane, and DDT were the most widely used pesticides in Egypt through the early 1980's. The pesticides are dangerous as they are very persistent in the environment and bioaccumulative in fatty tissue. In Egypt, chlorinated hydrocarbons (DDT) are still in use in some rural agricultural areas. Pesticide food contamination may be a major health threat to the general population in Egypt. Varying amounts of pesticides applied to crops in the field may remain on food surfaces or be incorporated systemically into the plant. Subsequent washing, processing, and cooking may remove some but not all of the pesticide residue. Pesticides may apply to crops after harvest (DDT) to prevent spoilage during transport and storage. Pesticides may even appear in crops to which they were not applied when irrigation water that has been contaminated by upstream pesticide use is re-used for additional crops.

\section{Power lines}

Power lines are surrounding the outlines of Cairo. There are power lines in Almarg in the north, in Helwan and Madii in the south, and in Mokatem and Naser city in the west as seen in figure1.

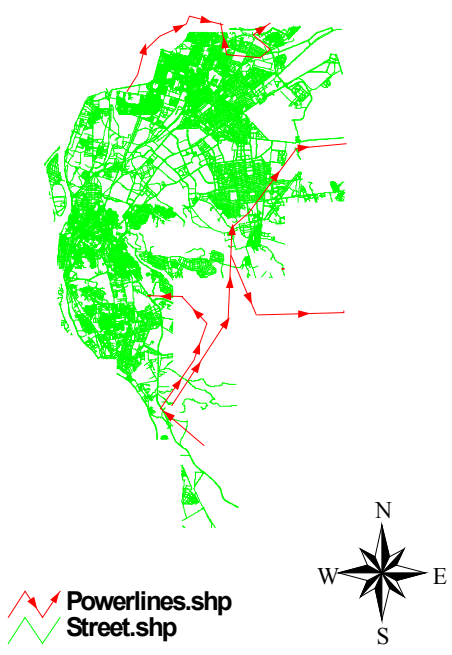

Figure 1. Power lines in Cairo

\section{Power stations}

The main regulation for Cairo Electricity Distribution Company has been issued and its fourth element stated that the geographic area for the company's activities includes Cairo, Giza, and the extension of Great Cairo in Kaloubia governorate. Due to the large size of this geographic area and overpopulation in the above mentioned governorates, a huge burden is put on the company in supplying the power services to the citizens in the governorates and in securing the feeding of electricity for different usages, through a large distribution network covering all cities and villages in the mentioned governorates serving a large number of citizens. This is clear when comparing the components of this network, the number of customers and the total number of assets and debits, with other distribution companies.

Power Stations were in four areas in Cairo (El Zawia, Al Sabtia, Naser city, Wadi hof).

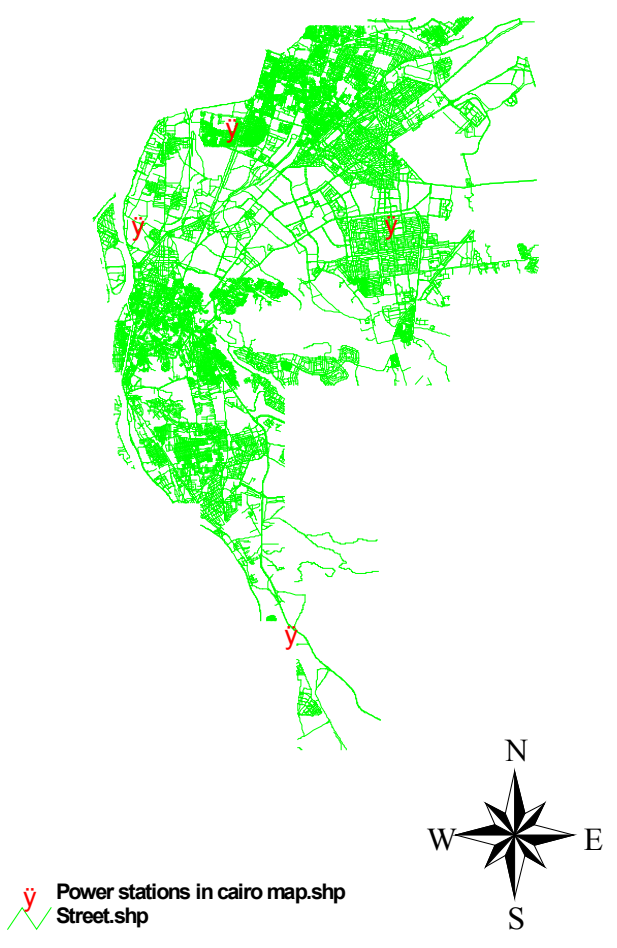

Figure 2. Power stations in Cairo

The concentration of leukaemia was in (Helwan, Masara, and Shoubra). So there is a positive correlation between leukemia and power stations. For Helwan and Massara there are one power station in Wadi Hofi. For Shoubra located between two power stations in Cairo ( North Cairo power station and Tebeen power station in Sabtia) Also in the nearest with Shoubra AlKamia power station.

\section{Effects of Industrial Pollution on Health}

The environment in which we live can be considered as having three fundamental sets of components: Physical (energy of one form or another), Chemical (i.e. substances whether natural or man- made), Biological (living things). Hazards can present themselves to us in various media e.g. air, water and soil. The influence they can exert on human health is very complex and may be modulated by our genetic make up, psychological factors and by the perceptions of the risks that they present. The cause and development of nearly every human disease is in so me way related to environ mental factors. Diet and nutrition, infectious agents, toxic chemicals, physical factors and physiological stress all play a role in the onset or progress of human diseases[15]. One of the 
characteristics of chemical or physical carcinogenes is is the usually extended period of time (latent period) between contact with the carcinogen and the appearance of a tumor. The latent periods of occupational cancers may extend from one to several years and commonly to several decades. Initiation and promotion are two stages in the development of tumors. Initiation is caused by chemical, physical, or biological agents, which irreversibly and heritable alter the cell genome[16].

\section{Modeling a Spatial Dss}

Modern SDSS such a system contains data and analysis functions of GIS, RS, DSS, and models and depends on given expert knowledge. To generate a SDSS with these topics, it is essential to implement a Model base Management System (MBMS) as well as a Database Management System(DBMS). By applying modern DSS by using GIS analysis functions (industrial pollution maps in Cairo and the distribution of some diseases), MBMS and DBMS to find the relations between some industrial pollution and some diseases. The modern SDSS can be help for landscape of industrial pollution which may be effect on the human health in Cairo, Egypt.

The spatial decision support system (SDSS) contains three main parts. The first are Geographic Information System (GIS) and Remote Sensing (RS), second is Decision Support System (DSS) Models, third are Expert System (ES) and Artificial Intelligence (AI). In order to building three databases geo-database, model database and knowledge database as in figure 3. This spatial decision support system (SDSS) can be used by decision makers for designing landscape in Egypt.

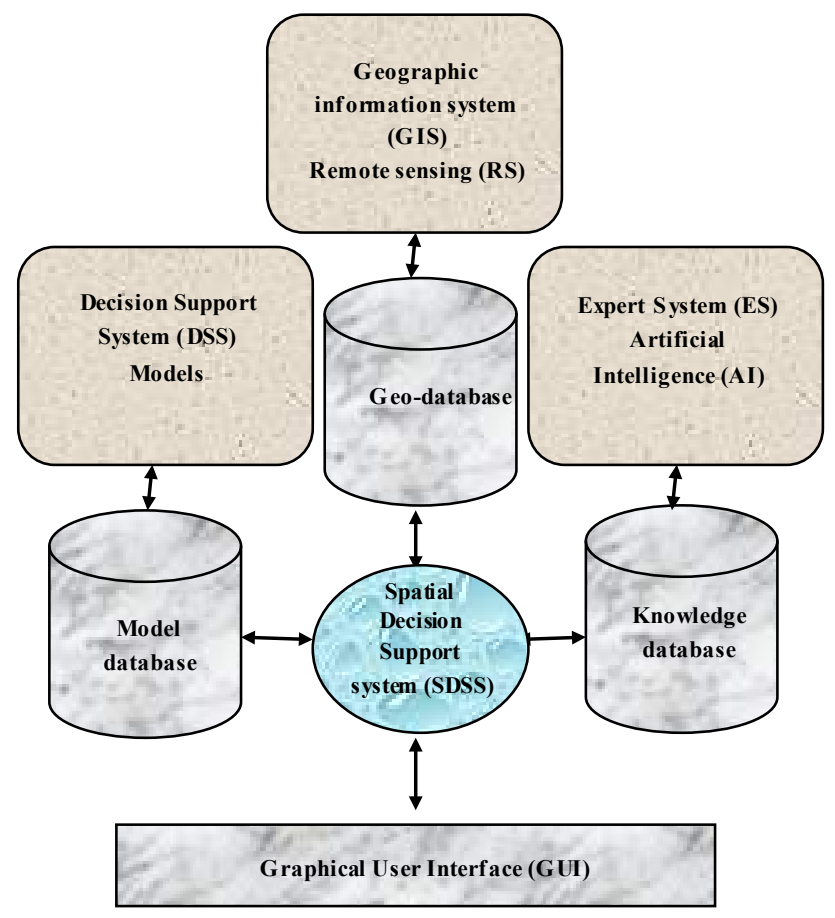

Figure 3. Spatial Decision Support system (SDSS)

\section{Conclustions}

Given the advances in computer technology in general and GIS techniques in particular, I suggest that SDS S will be an important component of DSS applications in future. This trend will be driven by the relevance of spatial in formation as a component of the information needed for a wide range of decisions. This class of DSS will make an important contribution, not because of its use of the latest technology, but because it will allow decision makers incorporate a spatial dimension in their decision making. This spatial dimension is an important feature of many areas of DSS application. These potential areas of application including fields, such as routing or marketing, which have been important fields of DSS application in the past. For this larger class of decision makers who might use SDSS, spatial data will be used with other types of information required originating in specialised models, often of a non-spatial nature. For this broad range of applications, GIS technology alone can only make a partial contribution to decision support. Comprehensive decision support will require the effective integration of GIS and non GIS techniques. This can be achieved by building systems using a GIS as a DSS generator. The building of SDSS by using GIS has been facilitated by recent technical developments, both within GIS software, and in programming tools generally.

\section{REFERENCES}

[1] "Geographic Information Systems as an Integrating Technology: Context, Concepts, and Definitions". ESRI. Retrieved 9 June 2011.

[2] Goodchild, Michael F (2010). "Twenty years of progress: GIScience in 2010". Journal of Spatial Information Science. DOI:10.5311/JOSIS.2010.1.2.

[3] Smajgl A, Morris S, Heckbert S. (2009). Water policy impact assessment - combining modelling techniques in the Great Barrier Reef region. Water Policy Journal 11, 191-202.

[4] van Delden, H. (2009). Integration of socio-economic and bio-physical models to support sustainable development. Presented at 18th World IMACS / MODSIM Congress, Cairns, Australia, 13-17 July 2009.

[5] A. Daash, A. Srivastava, B.N.Nagpat, R.Saxena and S.Kcipta, "Geographical Information System (GIS) in decision support to control Malaria- A case study of Korapat district in Orissa, India, "Journal of Vector Borne Diseases, Vol. 46 no 1, PP.72-74, 2009.

[6] Aggarwal, A.K., (2001). A Taxonomy of Sequential Decision Support Systems, University of Baltimore, USA, $\mathrm{http}: / /$ www.informingscience.org/proceed ings/IS2001Procee dings/pdf.aggarwalEBKAtaxa.pdf)

[7] Densham, P. J. and Goodchild, M. F. 1989, Spatial Decision Support System: a research agenda, Proceedings of GIS/LIS'89, ACSM, 707-716.

[8] Turban, E., et.al., Decision Support and Business Intelligence 
Systems. Eighth Edition, Pearson Educational, Upper Saddle River, 2007.

[9] Greene, R.; Devillers, R.; Luther, J.E.; Eddy, B.G. (2011) "GIS-based multi-criteria analysis". Geography Compass 5/6: 412-432.

[10] Egyptian Environmental Affairs Agency (MSEA/EEAA, 2001, Annual Report 2000/2001.

[11] European Commission, Compilation of Environment Information on Egypt, 1999.

[12] Ministry of Public Works and Water Resources, National Research Center, State of the Environment, Working Group A3, 1996.
[13] Egyptian Environmental Affairs (MSEA/EEAA, 2000, Annual Report 1999-2000.

[14] Central Agency for Public Mobilization and Statistics (CAPMAS), annual book, 2005.

[15] Feychting M, Pluto N, Nise G, Ahlboni A. Parental occup ational exp osures and childhood cancer. Environ Health Perspect 109(2), 2001.

[16] McMichael AJ., The role of epidemiologists in eradicability of poverty, 1998. 